\title{
Concurrent cases of bilateral anterior shoulder dislocation: our observations in three cases
}

\author{
Orhan Akinci', Yavuz Akalin², Ali Otuzbir², Alpaslan Oztürk² \\ ${ }^{1}$ Department of Orthopedics and Traumatology, Torbali State Hospital, Izmir, Turkey \\ ${ }^{2}$ Department of Orthopedics and Traumatology, Bursa Yuksek Ihtisas Training and Research Hospital, Bursa, Turkey
}

\begin{abstract}
Bilateral anterior shoulder dislocations are rarely seen and usually occur due to the same mechanism arising from traumatic injuries. In here, we discussed three cases of traumatic bilateral anterior shoulder dislocation, one had an additional patella fracture, and the other one had bilateral tuberculum majus fracture. All of the patients were female and our first case that presented here was 65-year-old and given a closed reduction for isolated bilateral anterior shoulder dislocation that occurred as a result of falling due to an epileptic seizure. The second patient was 70-year-old and had bilateral shoulder dislocation and patella fracture (right leg) after of falling down the stairs due to syncope. Closed reduction was applied to the bilateral shoulder dislocation. Patella fracture on the right leg was fixated using open reduction internal fixation method. The third patient, who was 57-year-old, fell down from the stairs and had bilateral anterior shoulder dislocation accompanied by bilateral tuberculum majus fracture, and she was given closed reduction. For all three of the cases, Hippocrates method was used as the closed reduction method.
\end{abstract}

Eur Res $J$ 2016;2(1):80-84

Keywords: Bilateral, dislocation, shoulder, traumatic

\section{Introduction}

Bilateral anterior shoulder dislocations are quite rare. They frequently occur due to trauma. Three patients who had bilateral anterior shoulder dislocation- one because of falling off a chair and the other two down the stairs, were evaluated in company with the related literature. Unilateral anterior shoulder dislocations are the most frequent large joint dislocations coming to emergency services. In contrast, bilateral anterior shoulder dislocations are quite rare. It could be an isolated dislocation while it could also be accompanied by shoulder fractures and fractures in other areas. A majority of bilateral shoulder dislocations occur in form of posterior shoulder dislocations, and the dislocations of the recorded cases occurred as a result of grand mal type epileptic seizures, electric shocks, and frequent contractions seen after electric shocks and hypoglycemia or voluntarily in psychic (mental) illnesses [1-6]. Anterior shoulder dislocations occur with extreme extension, abduction and external rotation force [7]. Since the forces causing dislocation have to affect both joints in a similar mechanism concurrently, they occur quite rarely. Therefore, they can easily be ignored $[8,9]$. 


\section{Case Presentations}

\section{Case 1}

A-65-year-old female falls down because of an epileptic seizure she had when trying to sit on a chair at home. In the medical examination, epaulette and motion restriction were observed. Neurovascular examination was natural. The graph of the patient showed a bilateral isolated anterior shoulder dislocation (Figure 1). Both dislocations were reduced closely with Hippocrates manoeuvre under sedation. In the control graphs, anatomic reduction was observed (Figure 2). Both shoulders were made immobile for three weeks using velpau bandage. Afterwards, they were included in a rehabilitation program. In the examination 16 months later, shoulder joints were observed to be stable and no motion restriction was found. The dislocation did not recur in the meantime.

\section{Case 2}

Seventy years old female patient fell down and rolling from the stairs as a result of a sudden syncope. Subsequently, she was taken to emergency service with pain in her shoulders, motion restriction as well as pain and swelling in the right knee. In the physical

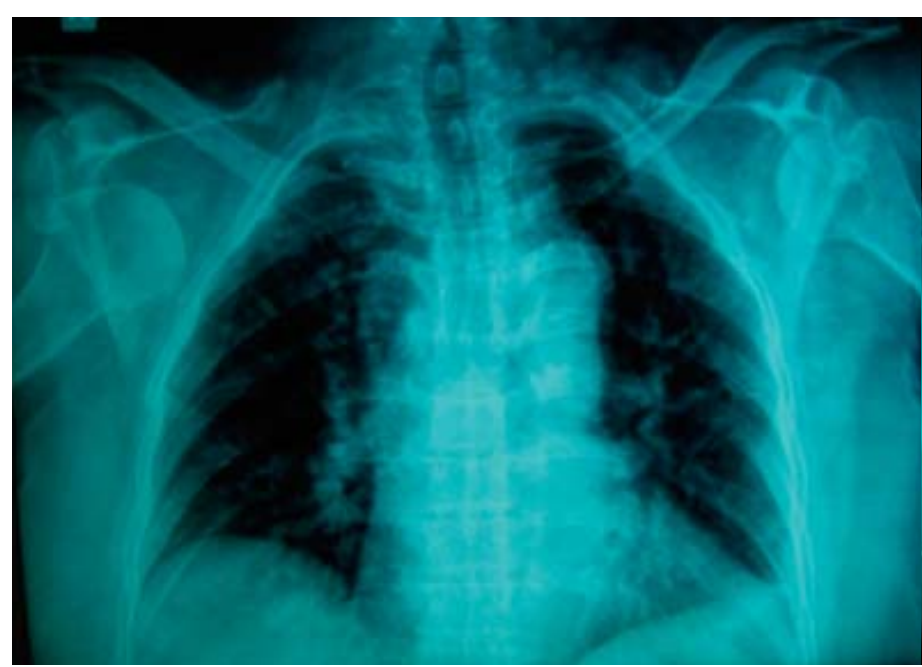

Figure 1. Case 1- Bilateral anterior shoulder dislocation radiograph

examination showed mild abduction and external rotation in both shoulders, findings of epaulette and motion restriction. The neurovascular examination was unremarkable. A swelling in the right knee, pain with palpation and patella deformity were observed. Graphs showed isolated anterior dislocation for both

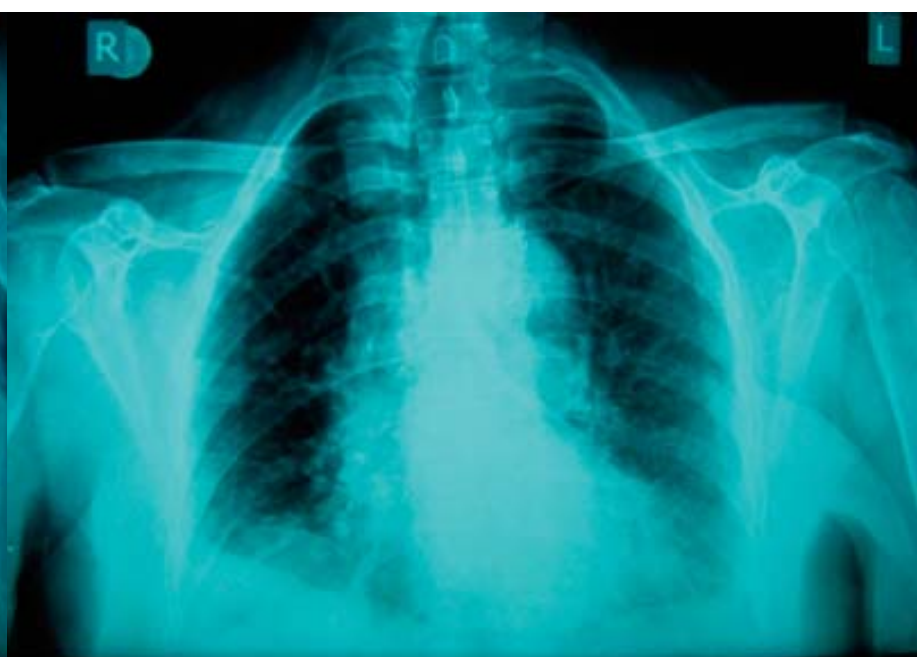

Figure 2. Case 1 radiograph after closed reduction

shoulders (Figure 3). She was taken into operation under emergency circumstances, and a closed reduction was performed on both shoulders using Hippocrates manoeuvre under general anaesthesia. Control graphs were taken (Figure 4). Both shoulders were immobilized for three months with an arm sling.

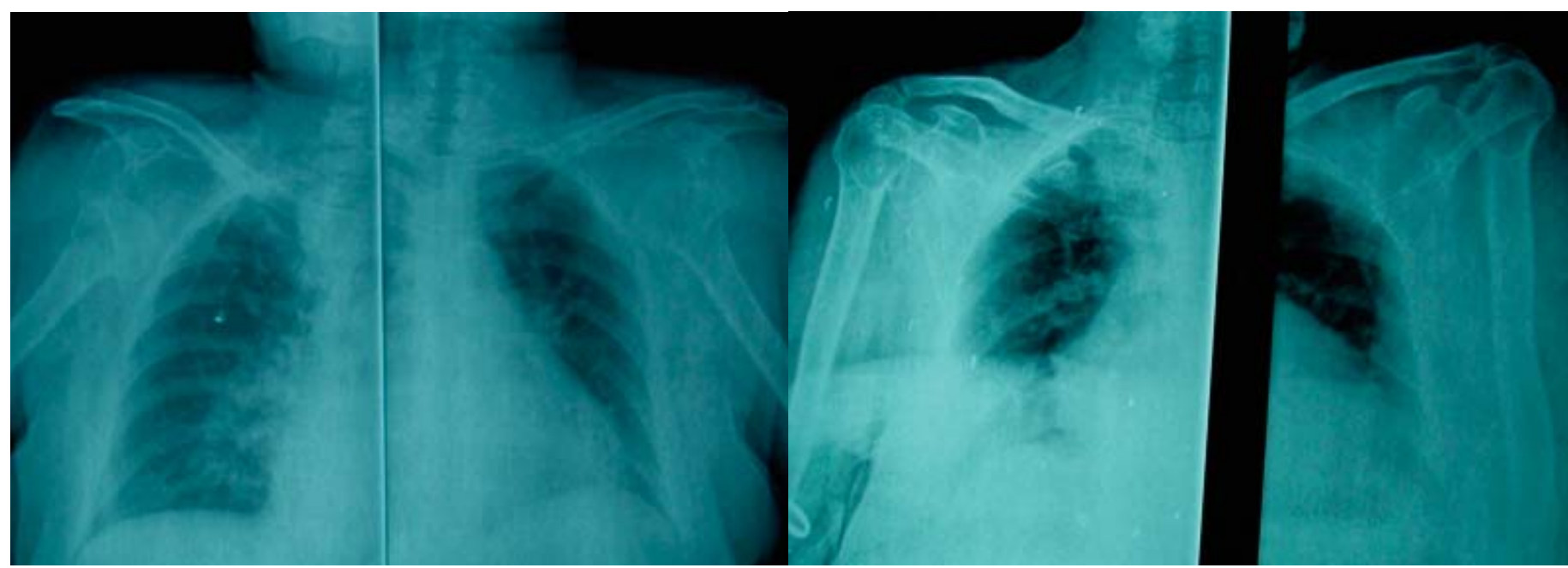

Figure 3. Case 2- Bilateral anterior shoulder dislocation radiograph
Figure 4. Case 2 - Bilateral shoulder radiograph after closed reduction 
Afterwards, these received physical treatment and rehabilitation for four weeks. In the controls 16 months later, it was seen that union in the patella was full, movements of the left shoulder were close to perfect, and there were insignificant minimal restrictions in external rotation and abduction of the right shoulder.and abduction of the right shoulder.

\section{Case 3}

A 53-year-old female patient admitted to the emergency service because of losing balance and falling when going down the stairs. She described to have fallen into open hands with both elbows in extension and rolled down 2 or 3 steps. In the physical examination, bilateral shoulder joint was deformed (epaulette sign) and the shoulder was observed to be in the mild abduction and external rotation.

Radiographs showed it was accompanied by a tuberculum majus fracture, and there was bilateral

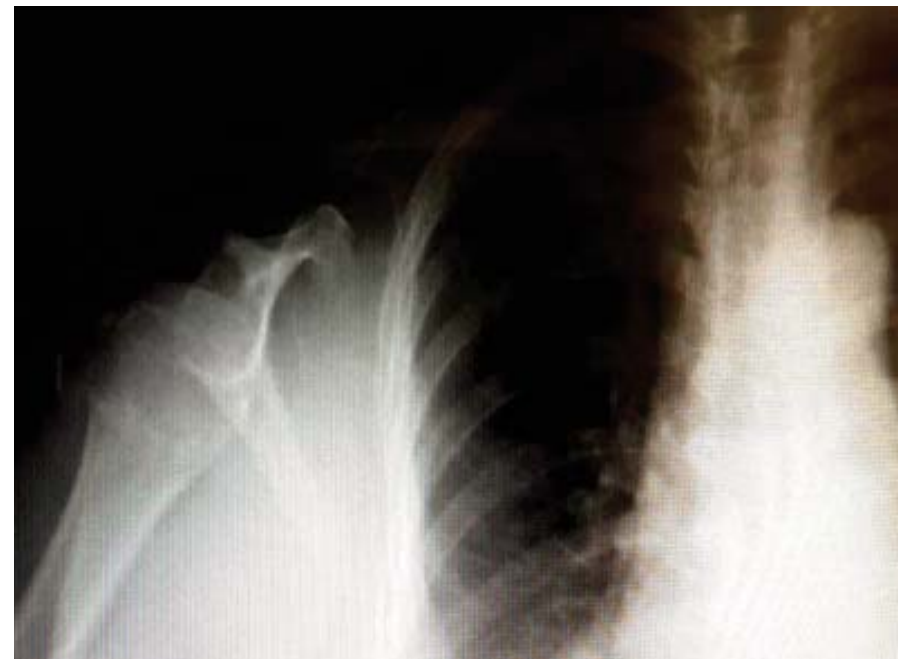

Figure 5. Case 3 -Bilateral shoulder dislocation right shoulder radiograph anterior shoulder dislocation. However, shoulder graphs of the patient before reduction were not taken on the same cassette, so we had no chance to repeat (Figure 5, 6). Under general anaesthesia, closed reduction was performed using Hippocrates method. Both shoulders of the patient were immobilized for three months with velpau bandage. Later, she was included in the physical treatment program. In the controls 16 months later, it was seen that bilateral shoulders movements were close to perfect, and that union and reduction were complete (Figure 7).

\section{Discussion}

Glenohumeral dislocations are the most commonly observed ones among all large joint dislocations. They occur as a result of trauma in many young men. It is followed by dislocation in elderly

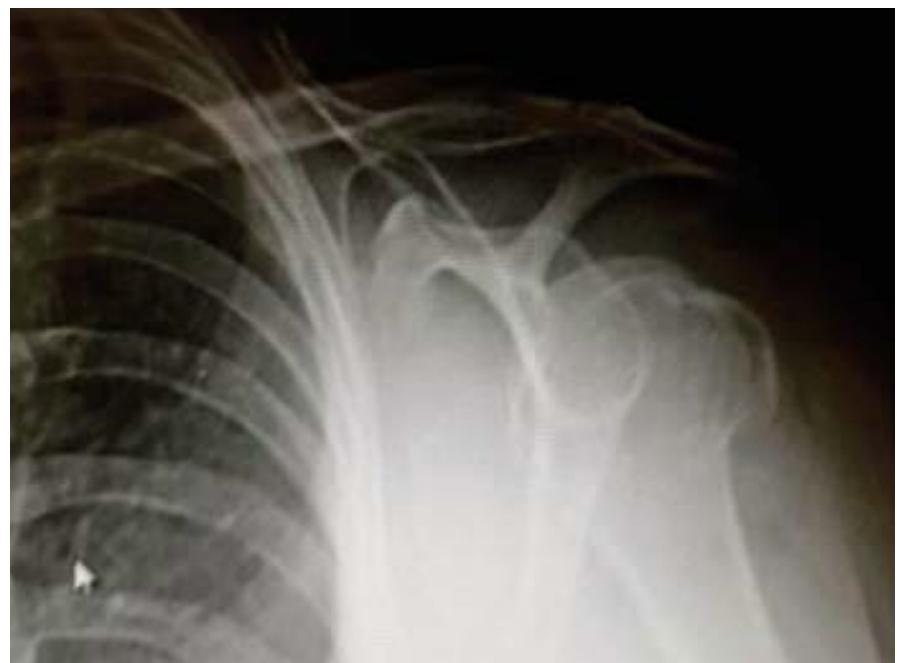

Figure 6. Case 3- Bilateral shoulder dislocation left shoulder radiograph

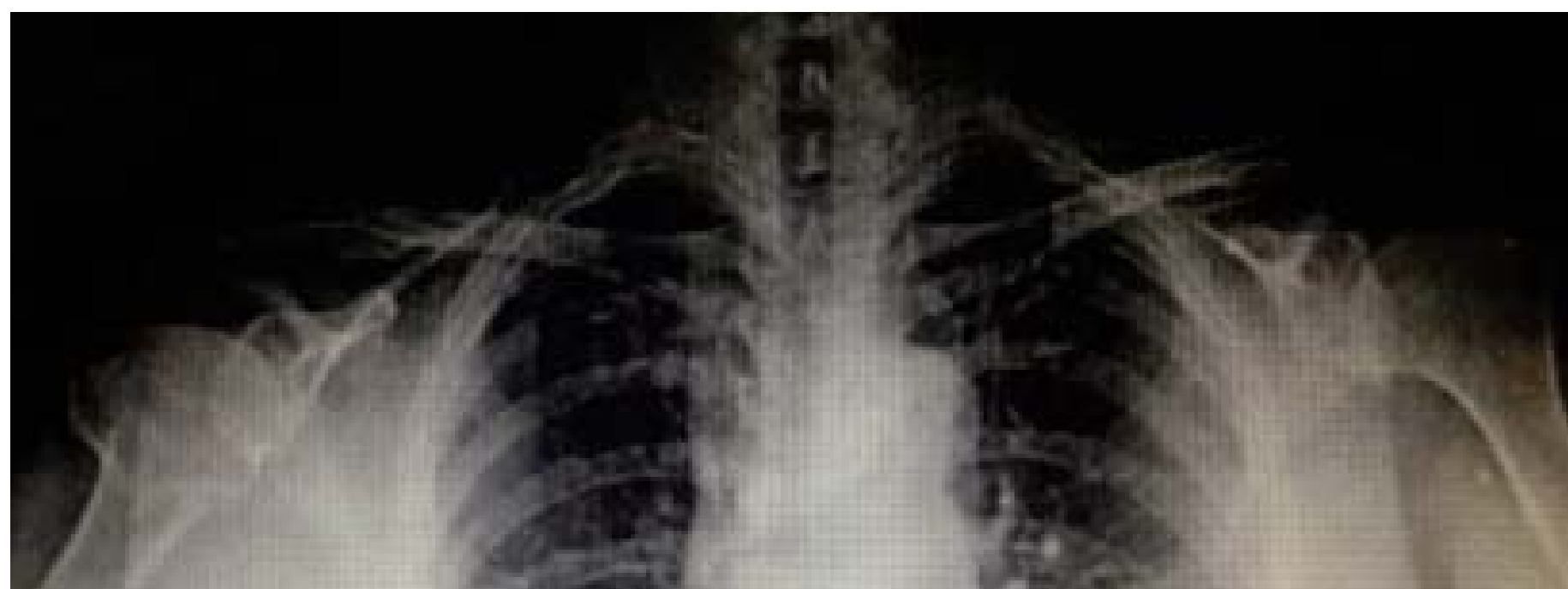

Figure 7. Case 3-Bilateral shoulder dislocation and tuberculum majus fracture radiograph after closed reduction 
women which occur due to the decrease in the number of crosslinks of collagen in the joint capsule and the increased risk of falling [10]. Bilateral shoulder dislocations occur rarely because of rotator muscles (M. infraspinatus and M. teres minor), which are weaker than the powerful internal rotator cuffs (M. latissimus dorsi, $\mathrm{M}$. pectoralis major and $\mathrm{M}$. subscapularis), dislocations frequently occur towards the posterior. Posterior shoulder dislocations most frequently occur as a result of grand mal type epileptic seizures, electric shocks, and frequent contractions seen after seizures due to alcohol deprivation and hypoglycaemia or voluntarily in psychic (mental) illnesses [1-6]. Contrary to posterior shoulder dislocations, bilateral anterior shoulder dislocations are seen very rarely, they usually occur following trauma.

Ozan et al. [12] reported 80 cases in the literature they review covering the period between 1902 and 2013. These dislocations have most often been accompanied by muscle tears, tuberculum majus or glenoid fractures and neurovascular complications [9, 11-15]. In the literature, among the etiological reasons of bilateral anterior shoulder dislocations, the rate of those occurrence caused by falls or strong extremity tractions is $50 \%$, those occurring due to convulsive crises, electric shocks strong muscle contractions including physical exercises is $40 \%$; and the rate of any cases other than trauma. such as neuromuscular diseases, hyperlaxity, and voluntary dislocations, is $10 \%[6,11]$.

Anterior shoulder dislocation mechanisms can be of various nature. They mainly occur combined with abduction, extension and external rotation forcing. Movements such as falls on open hand at elbow extension fall at shoulder extension, abduction or internal rotation, pivot effect of acromion at hyperabduction position and traction cause shoulder displacement $[6,16,17]$. Since two of our cases had fallen because of an epileptic seizure and syncope, they could not recall the form and mechanism of the falls. We believe that this trauma mechanism resulted from falling on open hand at elbow extension as stated in the literature. The fact that the third and the last case was conscious when falling down the stairs and rolled down with two hands open at elbow extension supports the possibility that other cases had occurred in a similar mechanism as well. It was reported that $15 \%$ of these patients are accompanied by tuberculum majus fracture [18], 30\% of those over 40 [19], and $54 \%$ of those older are accompanied by $54 \%$ rotator cuff tears [20]. No glenoid or tuberculum fracture and cuff tears were observed to be accompanying shoulder dislocation in two of our cases. In the third case, a more distinct bilateral tuberculum majus fracture accompanied the dislocation. After early reduction and immobilization, there is a need for passive and active physiotherapy to be given efficiently [10]. For patients whose pain cannot be relieved and sufficient range of motion cannot be obtained despite rehabilitation, rotator cuff tear must be considered, and an MRI image must be taken. Since sufficient range of the movement was attained in our cases after rehabilitation, MRI was not taken. Formation of a fracture along with the dislocation is associated significantly because the patient was over 40 , the dislocation occurred for the first time with the decreased bone density, ligamentous anatomy was strong, and it had occurred due to high energy trauma [21].

Especially after anterior displacement, there might be complications such as fractures, brachial plexus injury, vascular injury, soft tissue injuries and recurrent displacements. The possibility of recurrence after the first displacement is high in young patients. Our cases did not describe any prior shoulder displacement.

Another significant problem for these patients is the delay in the process of diagnosis. Dunlop [10] expressed more than $10 \%$ of them received delayed diagnosis. When patients apply to the emergency service following a seizure, very often they cannot remember the event in its entirety and complain most about the shoulder with pain. Unless careful examination is conducted, the dislocation of the other shoulder may remain undetected. Although it is the primary choice for treatment to prevent post shoulder dislocation recurrences, the priority is given to the treatment of complex structural injuries in elderly patients. The risk of rotator cuff injuries increases with the shoulder displacement at advanced age [18]. Even though the treatment of rotator cuff tears in these patients is controversial, first conservative treatment is given [20].

Post reduction fixation is usually done using Velpo bandage. Since Velpo bandage affects daily activities negatively in bilateral displacements, arm sling can be preferred. We fixated our first patient, who fell down due to a seizure of Grand mal epilepsy against the risk of having another seizure, with velpo bandage and the third patient due to bilateral tuberculum majus fracture; but we fixated the second patient with an arm sling which partly allows for shoulder movement. In 
the literature, Kocher manoeuvre is usually preferred as the reduction method $[3,4,6,9,16,18]$. Because of their elder age, we used Hippocrates manoeuvre for the reduction of the first two of our patients. It is because of the fracture risk during Kocher manoeuvre in osteoporotic patients. In the relatively younger third case, on the other hand, Hippocrates manoeuvre was used because of the tuberculum majus fracture. No serious complication was encountered in these three cases.

\section{Conclusions}

Although concurrent bilateral anterior shoulder displacements occur rarely, they can be seen about trauma in elderly patients; therefore, the neurovascular examination should not be ignored, and the possibility of fractures must be remembered.

\section{Informed Consent}

Written informed consent was obtained from the patient that was presented in this case.

\section{Conflict of interest}

The authors declared that there are no potential conflicts of interest with respect to the research, authorship, and/or publication of this article.

\section{References}

[1] Mancini GB, Lazzeri S. Bilateral posterior fracture-dislocation of the shoulder. Orthopedics. 2002 Apr;25(4):433-4.

[2] Songur M, Sahin E, Kalem M, Zehir S. Amputation for upper extremity ischemia following shoulder dislocation: case report and a review of literature. Eur Res J. 2015 Jul;1(2):66-70.

[3] Parrish GA, Skiendzielewski JJ. Bilateral posterior fracture- dislocations of the shoulder after convulsive status epilepticus. Ann Emerg Med. 1985 Mar;14(3):264-6.

[4] Prillaman HA, Thompson RC Jr. Bilateral posterior fracturedislocation of the shoulder: A case report. J Bone Joint Surg Am. 1969 Dec;51(8):1627-30.

[5] Fullarton GM, MacEvan CJ. Bilateral posterior dislocation of the shoulder. Injury. 1985 May;16(6):428-9.

[6] Botha AH, du Toit AB. Bilateral anterior shoulder dislocation: a case report of this rare entity. SA Orthop J. 2010;9(4):68-70.

[7] Galanakos S, Christodoulou E, Panayi C, Papadakis AS, Nicolaides V, Macheras GA. Bilateral anterior glenohumeral dislocation: A case report and a review of the literature. EEXOT. 2008;59(4):252-6.

[8] Hartney-Velazco K, Velazco A, Fleming LL. Bilateral anterior dislocations of the shoulder. South Med J. 1984;77(10):1340-1.

[9] Cresswell TR, Smith RB. Bilateral anterior shoulder dislocations in bench pressing: An unusual cause. Br J Sports Med. 1998 Mar;32(1):712.

[10] Dunlop CC. Bilateral anterior shoulder dislocation - a case report and review of the literature. Acta Orthop Belg. 2002 Apr;68(2):168-70.

[11] Fung DA, Menkowitz M, Chern K. Asymmetric bilateral shoulder dislocation involving a luxatio erecta dislocation. Am J Orthop (Belle Mead NJ). 2008 May;37(5):E97-8.

[12] Ozan F, Altay T, Koyuncu S, Celiker H. Bilateral anterior shoulder dislocation: A case report and a review of the literatüre. Ege J Med. 2014;53(1):56-59.

[13] Mehta MP, Kottamasu SR. Anterior dislocation of the shoulders with bilateral brachial plexus injury. Ann Emerg Med. 1989 May;18(5):58991.

[14] Noczynski L, Pawlowski A, Makowski Z, Lubowicz B. Bilateral anterior dislocation of the shoulder with vascular and neurologic sequelae. Chir Narzadow Ruchu Ortop Pol. 1973;38(1):31-3.

[15] Sharma L, Pankaj A, Kumar V, Malhotra R, Bhan S. Bilateral anterior dislocation of the shoulders with proximal humeral fractures: a case report. J Orthop Surg (Hong Kong). 2005 Dec;13(3):303-6.

[16] Ozer H, Baltaci G, Selek H, Turanli S. Opposite-direction bilateral fracture dislocation of the shoulders after an electric shock. Arch Orthop Trauma Surg. 2005 Sep;125(7):499-502.

[17] Cottias P, le Bellec Y, Jeanrot C, Imbert P, Huten D, Masmejean EH. Fractured coracoid with anterior shoulder dislocation and greater tuberosity fracture: report of a bilateral case. Acta Orthop Scand. 2000 Feb;71(1):95-7.

[18] Galois L, Traversari R, Girard D, Mainard D, Delagoutte JP. Asymmetrical bilateral shoulder dislocation. SICOT. 2003 Feb;E024:1-5. [19] Segal D, Yablon IG, Lynch JJ, Jones RP. Acute bilateral anterior dislocation of the shoulders. Clin Orthop Relat Res. 1979 May;140:21-2. [20] McFie J. Bilateral anterior dislocation of the shoulders: A case report. Injury. 1976 Aug;8(1):67-9.

[21] Ferkel RD, Hedley AK, Eckardt JJ. Anterior fracture-dislocations of the shoulder: pitfalls in treatment. J Trauma. 1984 Apr;24(4):363-7. 